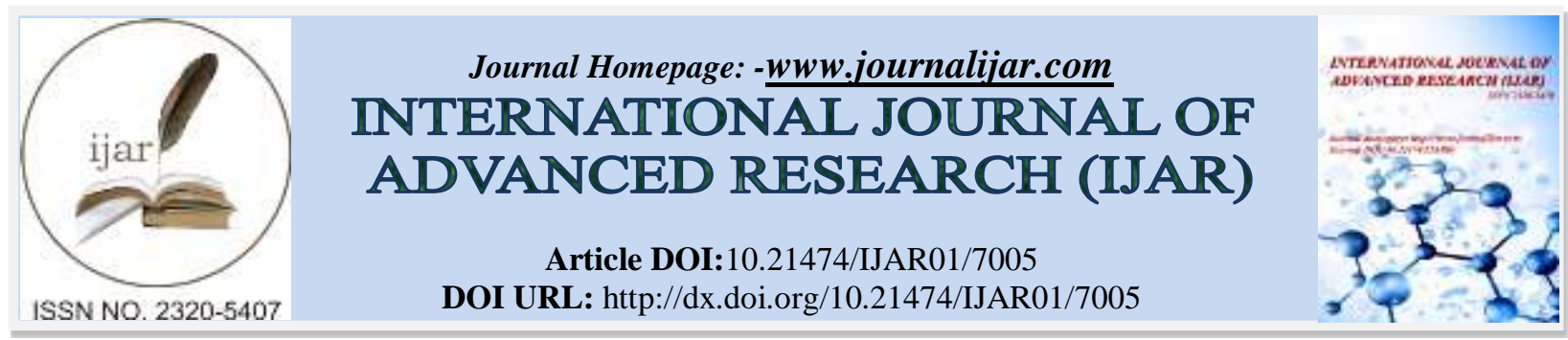

RESEARCH ARTICLE

\title{
CUTANEOUS MALIGNANT MELANOMAS: EPIDEMIOLOGICAL, CLINICAL AND THERAPEUTIC ASPECTS. EXPERIENCE OF THE PLASTIC SURGERY DEPARTMENT IN MOHAMMED VI HOSPITAL OF MARRAKECH- MOROCCO.
}

\author{
Ouafaa Dhaidah, Mahdi Sahibi, Abdelkodus Bhihi, Mariem Quaboul, Adil Dehhaze, Moulay Driss Elamrani, \\ Yassine Benchamkha and Salwa Ettalbi. \\ Department of Plastic and Reconstructive Surgery in CHU Mohamed VI, Marrakech, Morocco.
}

\section{Manuscript Info}

Manuscript History

Received: 01 March 2018

Final Accepted: 03 April 2018

Published: May 2018

Keywords:-

melanoma, cancer, dermatology.

\section{Abstract}

Melanoma is a tumor proliferation developed at the expenseof melanocytes, the incidence of melanomais increasingworldwidemakesita realpublic health concern, the severity of thismalignancy tumor isassociated witha strong ability tometastasize, involvingthe prognosisof the patientif the lesionis notdetected early henceimproving earlydiagnosis remainsa major challenge.

The objective of ourwork is to studythe epidemiological ,pathological, therapeutic, prognostic and preventive aspects of melanomathrough aretrspective studyof 33patientscollectedfrom January 2010 to January 2017 inin plasticsurgery andburnsofthe CHUMohammed VIMarrakech. The average age of our patients was 61 years with a female predominance. Sun exposure was found in $91 \%$ of our patients which supports the important role complained of sun exposure in the development of melanoma.

The location the sole of the foot is by far the most frequent ( $73 \%$ of our patients).

On histological level, Acrolentigineux (61\%) and nodule (24\%) types are the most common, with a Breslow thickness varies between $2 \mathrm{~mm}$ and $12 \mathrm{~mm}$ and Clark level varies between IV and V in $75 \%$ of our patients, reflecting the significant delay between the start of the lesion and the consultation which is estimated at 2 years and consequently the lack of effective means of screening for patients at risk in our population.

On therapeutically level, at the initial stage, only surgical excision, constituting the gesture that allows both diagnosis and treatment of the lesion which must be done as soon as possible to ensure prolonged survival.

At the loco-regional stage, surgical resection with regional lymph node dissection is the standard of care. The sentinel node biopsy provides very precise classification of cutaneous melanoma, but has not been reported to have a significant impact on survival. The dissection effect on survival is a subject of inquiry.

Two distinct approaches have emerged to try to prolong the survival of patients with metastatic melanoma: Immunomodulation with anti- 
CTLA4 monoclonal antibodies and targeted therapy with inhibitors of BRAF or MEK for melanoma with BRAF mutated, recent studies have shown that the combination of inhibitors of BRAF and inhibitors of MEK may improve the progression-free survival and eventually increase overall survival, the choice of therapy should be individualized in each case depending on various factors such as BRAF mutation status, age, co-morbidities and topography of metastases ,while chemotherapy and radiation therapy can be used to relieve the symptoms without significant impact on patient survival.

Preventing melanoma is a real public health problem requires education of the general population on the need for sunscreen and awareness of melanoma and encouraging early diagnosis with the training of health professionals.

Copy Right, IJAR, 2018,. All rights reserved.

\section{Introduction:-}

Melanoma is a malignant tumor developed at the expense of melanocytes. $90 \%$ of these tumors are cutaneous melanomas that arise from the melanocytic cells of the skin.

Melanoma is classically regarded as a rare tumor, but currently all statistical studies agree for a steady increase in frequency over the last two decades in all parts of the world, particularly in countries with strong sunshine.

Melanoma is still the subject of several studies because of its seriousness, its clinical polymorphism and especially its therapeutic difficulties.

The prognosis of melanoma remains dark, given its significant metastatic potential. He rests essentially on anatomopathological data, therefore, any suspicious cutaneous lesion of melanoma should be excised immediately without excision biopsy to establish the diagnosis at an earlier stage and therefore compatible with curative treatment.

This anatomopathological assessment of the prognosis remains the best guide to ask the therapeutic indication which includes, besides, surgery: first-line treatment, immunotherapy, chemotherapy and radiotherapy.

The objective of the present work is to analyze the epidemiological characteristics of malignant cutaneous melanomas for our population, to study its clinical and histological aspects and to take stock of the therapeutic care in its various stages.

\section{Material and methods:-}

This is a retrospective study was performed over seven years from January 2010 to January 2017 involving 33 patients at the Plastic Surgery, reconstruction and burns department of the Mohammed VI Hospital in Marrakech in Morocco. All our patients had an anatomopathological confirmation by the tumor lesion biopsy.

\section{Discussion:-}

Statistical studies confirm that the incidence of melanoma has been steadily increasing $[1,2]$.All over the world in recent years, particularly among the white population. Thus, the incidence of melanoma doubles every 10 years, and no country seems to be spared by the increase in the frequency of melanoma [3]. Skin melanomas make up 1.2\% of new cases of cancer in the world with a number estimated at 105,000 new cases per year. The highest rates are found in areas close to Ecuador such as: Australia, New Zealand and Hawaii [4]. In Europe, France has among European countries intermediate rates of incidence of melanoma ( 7 to 10 per 100,000 inhabitants per year), between high rates recorded in northern countries such as Norway (man: 14.3 per 100,000 inhabitants per year, woman: 16.1 per 100,000 inhabitants per year) and. This north-south gradient highlights the importance of phototype in the occurrence of melanoma [5]. In the United States of America, melanoma has an incidence rate that varies from 10 to 20 cases per 100,000 inhabitants per year [6]. In Africa, despite intense sunlight, countries have low rates, In Morocco, according to the study of S. El Mesbahi, 91 cases of cutaneous melanoma were recorded between 1984 and 2004 at IBN ROCHD CHU Casablanca giving an annual rate average of 4.33 cases per year [7]. Another study 
carried out at the IBN SINA CHU in Rabat between 1994 and 2005 reported an average annual rate of 2.5 cases per year with a marked increase in the incidence of melanoma during those years [8].

In our population, the annual average rate was 4.5 cases per year. There has been an increase in the number of cases of melanoma since more than $50 \%$ of our cases have been diagnosed in the last four years. These results are consistent with those of the literature.

The male predominance in our series is consistent with Moroccan statistical data. In a study of 287 melanoma cases in the Casablanca and Rabat Hospitals over a period of 22 years. Men are $61 \%$ compared to 39\% for women [5]. In Western countries, women are more frequently affected than men (Table I). This male predominance in our country is due to solar exposure, most probably related to the participation of men in working life, especially in rural areas.

\begin{tabular}{|c|c|c|}
\hline \multirow{2}{*}{} & \multicolumn{2}{|c|}{ Sex } \\
\cline { 2 - 3 } & Men & Women \\
\hline A. Bullet [6] & 40,7 & 57,3 \\
\hline N. M. Fisher [10] & 39 & 61 \\
\hline J. M. Halna [11] & 47 & 53 \\
\hline B. K. Armastrong [12] & 42 & 58 \\
\hline H. Thameur [8] & 63,33 & 36,67 \\
\hline F. Benouna Biaz [9] & 61 & 39 \\
\hline Our Population & 60 & 40 \\
\hline
\end{tabular}

Table I:-Comparison Between Sexes In The Different Populations In The Studies

According to the studies, The risk of melanoma increases with age (confirmed during studies of 60-years , but melanoma is not rare for people under the age 30 years. In fact, it is one of the most common cancers among young adults (especially young women). [13]

In the United Kingdom between 2009 and 2011, an average of $27 \%$ of cases were diagnosed in people under 50 years of age and an average of $24 \%$ of cases were diagnosed at the age of 75 years and over [14]. The average age of our patients is 61 years, which is consistent with the literature data.

The most common zone for melanoma for men is the upper back whereas in women, the most common sites are the legs [15].

The frequency distribution of melanoma by anatomical zone differs between Caucasians and ethnic minority populations, whereas Caucasians have a predilection for developing lesions on sun-exposed surfaces, including the face and neck, Blacks have lesions mainly located on the photo-protected mucosa and acute zones, particularly the foot, which is thought to be related to a traumatic lesion. In our series: the topography of lesions of patients in our study is shown schematically in Table II and It is noted that melanoma is localized in the lower limb (sole and toe) in $80 \%$ of cases, This predominance is explained by the fact that this zone constitutes a non-photo-protected part by the natural pigmentation and often victim of repeated microtrauma that would be involved in the genesis of melanoma.

Sun exposure is the main factor in the known environment associated with the development of skin cancer of all types. Although there is no standard measure, sun exposure has generally been classified as intermittent or chronic, and its effects can be considered short-term or cumulative.

Intermittent sun exposure appears to be the most important risk factor for melanoma [16,17]. Analytical epidemiological studies have shown the role of intermittent exposure to the sun and are shown schematically in the following table (with odds ratios [OR] of 1.6 to 1.7 )

A patient with a history of melanoma has a high risk of developing additional primary melanomas, especially for those with risk factors for melanoma, such as cutaneous phenotype, family history, mutation in the CDKN2A gene, exposure to the sun Intensive in young age, and many common nevi and / or atypical nevus, about 5\% of patients with melanoma develop more than one primary cancer, while in the family setting the corresponding estimate is $30 \%$, this rate of several primary cancers of the same organ is a common feature of hereditary cancer susceptibility 
syndromes and represents a clinical finding that should elevate the level of suspicion for a patient with melanoma and may be related to an underlying genetic predisposition. that was the risk of secondary melanoma after the diagnosis of a first prima melanoma ire is about $5 \%$ and is higher for men and elderly patients [29-30].

Another van der Leest cohort study [31] has shown that the risk of malignant melanoma is 8-15 times higher for people with a malignant melanoma history and that frequency and higher for women.

On the other hand, patients with a personal basal cell carcinoma or squamous cell carcinoma history is also associated with an increased the melanoma risk. According to studies [32-33], this risk varies from a non-significant increase for the melanoma with a squamous cell carcinoma history of 1.04 (CI 0.13-8.18) at a significant risk of 7.94 (CI 4.11-15.35).In our study, no history of malignant melanoma or skin cancer was documented.

In general, family history of melanoma increases the risk of melanoma by about the double; a family cancer registry study has evaluated more than 20,000 individuals with melanoma and found an incidence ratio of 2, 62 for the descendants of individuals with melanoma and 2.94 for siblings [34].

First-degree family members studies of 23,000 melanoma patients found a lifetime cumulative melanoma risk of $2.5 \%$ to $3 \%$, which is about twice the risk of the general population and when two or more Family members were diagnosed with melanoma before the age of 30 years and the cumulative lifetime risk for family members increased to $14 \%$ [34].

The gene for susceptibility to CDKN2A major hereditary melanoma is found to be mutated approximatively from $35 \%$ to $40 \%$ of families with three or more cases of melanoma. Up to date more than half of the families with multiple melanoma cases have no identified mutation [35].

The definition of a family group of melanoma varies by geographic regions around the world because of the role played by UV radiation for the pathogenesis of melanoma. In highly sunny regions, three or more affected family members are needed then that in areas with lower levels of ambient sun exposure, two or more affected family members are considered sufficient to define a family group. The American College of Medical Genetics and the National Society of Genetic Counseling recommend that a person with any of the following characteristics be referred for genetic counseling [36, 26].

A personal story of three or more primary melanomas.

Immunosuppressants (azathioprine and cyclosporine) are classified by the International Agency for Research on Cancer as causes of all types of skin cancer [37]. The risk of malignant melanoma is $80 \%$ higher for people with Crohn's disease [38]. On the other hand, the risk of malignant melanoma is $23 \%$ higher for people with ulcerative colitis [38]. Another study by Dinh [39] showed that melanoma is 1.6 to 2.5 times more common for organ transplant recipients than in the general population, an excess that has generally been attributed to the effects of cancer. Immunosuppressive therapy administered to prevent transplant rejection.

According to a study by Oslen [40] the risk of malignant melanoma is 50\% higher in case of HIV infection.

Some authors claim that the occurrence of acrolinogenic melanoma is $25-55 \%$ of cases due to trauma based on the fact that these melanomas occur in weight-bearing areas and their preponderance in thumb and fat nails toe who are more exposed to trauma [41].

The notion of trauma is currently controversial, in the retrospective study of Kaskel [42] only $8.7 \%$ (32 patients) of patients considered an association between trauma and melanoma formation. For these 32 patients, 22 patients reported only one event and the other ten have presented a persistent irritation and he concluded that on the epidemiological basis and clinical and scientific research up to date, there seems to be no evidence of the role of trauma in the pathogenesis of melanoma.

Focusing on our patients antecedents, we note the concept of foot trauma for a patient who presented a nodular melanoma of the sole of the foot while the concept of repetitive strain injury was found for two patients (farmers) who presented acrolentiinous melanoma of the foot sole. 
The risk of malignant melanoma is 2.2 times higher for aircraft pilots and flight attendants than for the general population, which can be explained by ultraviolet levels that are higher at altitude than at ground level[44].

Other occupational exposures have been variously and inconsistently associated with melanoma risk. If these reports are authentic, these exposures are likely to represent only a small fraction of cases [45].

The risk of melanoma is not associated with retinol consumption, however, case-control studies indicate a lower risk with increased consumption of retinol [46].

Other studies have shown an association between exposure to chemicals (such as Arsenic) and solvents and the occurrence of melanoma, but further studies are needed to support or refute this association.

A lesion likely to be a melanoma if these clinical criteria are present:

1. Asymmetry: Half of the lesion does not correspond to the other half

2. Irregular edges: The edges are ragged, notched or blurred.

3. Color variation: pigmentation is not uniform and can display shades of beige, brown, or black; white, red, blue.

4. Diameter: A diameter greater than $6 \mathrm{~mm}$ is characteristic, although some melanomas may be smaller; any growth in a nevus deserves an evaluation.

5. Evolution: Changes in the lesion over time are characteristic, this factor is essential for nodular melanoma or achromic (non-pigmented) melanoma, which may not have ABCD criteria above.

The ABCDE rule has a high diagnostic accuracy when used in combination, lesions with these characteristics (ABCDE) should be considered as a potential melanoma, although atypical /dysplastic nevi may be difficult to distinguish clinically. The more recent use of the ugly duckling sign may help for the detection of lesions that do not meet the classic $\mathrm{ABCDE}$ criteria (eg, nodular melanoma, achromic melanoma or desmoplastic melanoma).

In 1998, Grobet al [47] introduced the concept of ugly duckling based on the principle that when a "spot" is not like the others is often suspected of being malignant, this clinical realization emphasized the importance not only of evaluating the morphology of the lesion but also of comparing it to that of the surrounding lesions and looking for an aberrant value in the fundus of naevis having a similar appearance, this concept is based on the principle that any injury different from the others should be considered as a suspect naevus.

Dermatoscopy is a non-invasive method that allows in vivo evaluation of the colors and microstructures of the epidermis, the dermal-epidermal junction and the papillary dermis that is not visible to the naked eye. The identification of the specific diagnostic models is related to the distribution of colors and dermatoscopic structures that may indicate better the malignancy or benignity of a pigmented skin lesion.

According to the study done Vestergaard ME [48], that showed that dermatoscopic examination is more accurate than clinical evaluation with the naked eye for the diagnosis of cutaneous melanoma $(\mathrm{OR}=15.6, \mathrm{p}=0.016)$. In this study the mean sensitivity in the diagnosis of melanoma was $74 \%$ for the naked eye examination and $90 \%$ for the dermatoscope. However, the dermatoscopic examination is highly operator-dependent and is based on a color distribution analysis as a reflection of the malignancy and is therefore subject to the same pitfalls when the color reflects poorly or not the the tumor architectural disorder. In addition, the diagnosis of nodular or purely dermal pigmented tumors remains difficult with the dermoscopy whose field of vision extends essentially to the papillary dermis.

In our series, no patient has has done a dermatoscope exam.

Any suspect pigmented lesion should be sent to the biopsy. However, the method between a biopsy-excision and biopsy-simple has been the source of many studies.

The generally preferred method is the complete and full-thickness biopsy-excision of the suspect lesion with a small margin of normal-looking skin (approximately $3 \mathrm{~mm}$ ), this allows a reliable result on the histological nature and level. invasion. It is important to clearly document the dimensions of the lesion in the procedure of the physician, 
along with the dimensions of the lesion as well as the surgical margin, this will serve as a guide for the extra surgical margins that may be needed if a melanoma is diagnosed.

A simple biopsy of the lesion should not be performed in case of:

1. Lesion whose location makes complete excision difficult.

2. Nail lesion.

According to Balch CM [49], he concluded that in the case of small pigmented lesions, if an excisional biopsy can't be performed and that the entire lesion can be removed by punch-biopsy then the latter is an appropriate option. However, in case of a larger lesion the punch biopsy can't capture the malignant part and it may be necessary to perform a larger deep shave (shave-biopsy) at a part of the lesion where lies the most atypical pigmentation network.

In addition, according to Tran KT [50], the most important aspect of all these methods is to obtain an adequate sample that will allow a definitive diagnosis (including measuring the Breslow index) or excluding the diagnosis of melanoma by an experienced pathologist.

In general, many studies have demonstrated the superiority of biopsy-excision compared to other types of biopsy: shaved-biopsy and punch-biopsy. In fact, the rate of metastatic diffusion as well as the mortality rate are decreased for the patients who underwent a biopsy-excision compared to those who underwent other types of biopsies [51].

The performance of the pathology examination depends on the experience of the pathologist, the quality of the excisional material and the technique of preparation and study of the specimen.

In general, Broche has concluded that the pathological examination can invalidate or confirm the clinical diagnosis and provide essential prognostic elements to the clinician and that several elements are important for optimal performance of the pathological examination: experience of the pathologist, the quality of the biopsy-excision and the accuracy of the clinical data.

In our population, eight patients had nodular type melanoma (24\%), twenty patients had Acrolentiginous melanoma (61\%), two patients presented with SSM melanoma (6\%) and three melanoma cases with melanoma. of Dubreuil $(9 \%)$. These results are consistent with literature data and this high frequency of acro-lentiginous melanomas can be explained by the dark phototype (51\% with phototype IV and V) and plantar location (73\% of patients).

In our study, thoracic radiography is performed for 31 patients and abdominal ultrasound is performed for 7 patients. Echography of ganglion areas performed for 22 patients and the local radiography is performed for two patients. The thoraco-abdominal-pelvic CT scan was performed for sixteen patients.

A facial and brain scan was performed for one patient with melanoma in the cheek.

A cerebral CT scan was performed for one patient with melanoma of the scalp.

Whole body MRI is performed for one patient with melanoma of the sole of the foot.

This extension assessment performed for our patients revealed metastases for 13 patients (39\%):

1. one patient with brain metastases

2. 11 patients with Ganglionic metastases

3. Liver metastases in a patient.

The frequency of lymph node metastases at the time of diagnosis can be explained by:

1. The negligence of the patient's lesion especially that it is not accompanied by painful symptoms.

2. The high Breslow index (greater than $2 \mathrm{~mm}$ in $82 \%$ of cases).

3. Clark level as high at the time of diagnosis (at V for $27 \%$ and at IV for $48 \%$ of cases).

4. The relatively "hidden" location of certain lesions (for example at the plantar level in $73 \%$ of cases).

5. Financial constraints that prevent the public from consulting.

Surgical excision remains the main modality for the melanomas treatment, they are very curable in the localized stages and their treatment is mainly surgical excision with margins proportional to the microstage of the primary lesion in $80 \%$ of cases. [52] 


\begin{tabular}{|c|c|c|}
\hline Chronic intermittent Studies & Sun exposure OR*=95\%CI** & Sun exposure OR=95\%CI \\
\hline Nelmas al [18] & 1.6 & 0.7 \\
\hline Elwood al [19] & 1.7 & 0.9 \\
\hline Gandin al [20] & 1.6 & 0.9 \\
\hline
\end{tabular}

Table II:-Results Of Epidemiological Studies $[18,19,20]$ On Sun Exposure As A Risk Factor For Melanoma

$* \mathrm{OR}=$ odds ratio, $* * \mathrm{CI}=$ confidence interval;

Recently, the use of UV-emitting tanning devices is classified by the American Institute for Cancer Research as a cause of melanoma [21, 22]. According to these studies, 100 deaths from malignant melanoma each year in the UK are caused by the use of these tanning devices $[23,24]$.

Colantonio $\mathrm{S}$ [25] has shown that the risk of malignant melanoma is 16 to $25 \%$ higher for people who have ever used a tanning device (at any age) compared to those who have never used.

Boniol M [26] has shown that the risk of malignant melanoma is 59\% higher for people who have used these tanning devices before the age of 35 years.

For our population, the notion of medium intensity solar exposure was found for 30 patients (91\% of patients), which supports the important and incriminated role of sun exposure in the occurrence of melanoma.

The Fitzpatrick classification classifies individuals according to the reaction of their skin during solar exposure, pigmentary characteristics are determinants in the development of melanoma and there is an inverse correlation between the risk of melanoma and the color of the skin.

Scherer [27] has shown that dark-skinned ethnic groups (Blacks, Hispanics, darker Asians) have a very low risk of developing melanoma, however they develop melanoma on less pigmented, acral surfaces (sole). nail bed), the color of the skin is modified by genetics and behavior. MC1R is one of the main genes controlling pigmentation.

Olsen [28] has shown that the risk of malignant melanoma is doubled for people with phototype I compared to those with phototype IV.

Olsen [28] has also shown that the risk of malignant melanoma is doubled for phototype II and 35\% more for phototype III, compared with phototype IV.

In our study: 14 patients (42\%) had a group III phototype, 14 had a group IV phototype (42\%), 3 had a group V phototype (9\%), and 2 had a group II phototype ( $6 \%$ ).

These divergences of results with the literature can be explained by the important degree of sunshine in our population which explains the high occurrence of melanoma even in the dark phototypes and also by the predominance of the acrolentiginous melanomas (35\%).

Two analytical studies $[20,28]$ have shown that the risk of malignant melanoma is approximately 4 to 10 times higher for people with unusually shaped or large necroses (heterogeneous appearance, irregular margins). On the other hand, Gandini [20] has shown that malignant melanoma is nearly seven times higher for people with many (more than 100) common nevi compared to people with very little naevi (0-15 nevus) and that the risk of malignant melanoma increases by about $2 \%$ for each additional common naevi, so patients with multiple naevi demonstrate an increased risk of melanoma.

It is obvious that the presence of both multiple naevi and the presence of multiple clinically atypical nevi are associated with an increased risk of melanoma, most studies show a high risk of melanoma with the presence of atypical nevi.

In our study, seven patients presented with pre-existing melanoma of naevi, two of whom developed SSM-type melanoma, two patients with nodular melanoma and three patients with acrolentiginous melanoma, and three patients with melanoma on Dubreuil melanoma. 
The margins of lateral excisions are shown schematically in the following table:

\begin{tabular}{|l|l|}
\hline Breslow Indice $(\mathrm{mm})$ & margins of excision $(\mathrm{cm})$ \\
\hline In situ & $0,5-1$ \\
\hline$\leq 1$ & 1 \\
\hline 1,01 & $1-2$ \\
\hline $2,01-4$ & 2 \\
\hline
\end{tabular}

Table III:-Margin of lateral excisions

An excision margin greater than $3 \mathrm{~cm}$ is not recommended, as no significant benefit in terms of recurrence or survival has been demonstrated beyond [52].

The margins of deep excision, not specified in the standard options and recommendations and they are performed according to the anatomical topography and carrying all the subcutis.

In our population, 33 patients have performed a surgical excision with margins of lateral resection varying between $1 \mathrm{~cm}$ and $3 \mathrm{~cm}$, respecting the recommendations and the safety margins of the deep excision taking all the hypodermis.

Lymphatic Mapping and sentinel lymph node biopsy can be considered to evaluate the presence of occult metastases in regional lymph nodes of patients with primary tumors larger than 1 to $4 \mathrm{~mm}$ potentially identifying individuals that can be spared morbidity from the regional lymph node dissection and individuals who can take advantage of an adjuvant treatment.

Therefore, to ensure accurate identification of the sentinel lymph node, lymphatic mapping and sentinel node excision must precede by an extensive excision of the primary melanoma.

Several studies have demonstrated the diagnostic accuracy of sentinel node biopsy with false negative rates from $0 \%$ to $2 \%$.

If metastatic melanoma is detected by biopsy then a complete regional lymph node dissection can be performed in a second procedure.

For our population, ganglion dissection was performed for 19 patients (59\%):

1. 13 patients $(68 \%)$ underwent nodal lymph node dissection, of which 8 patients $(61.5 \%)$ reported metastatic lymph nodes

2. 6 patients (32\%) underwent lymph node dissection in principle (without palpable lymphadenopathy), 3 of which (50\% of the lymph node dissection performed) showed metastatic lymph nodes, which supports the important role of imaging and of the anatomopathological study in the research for occult lymph node metastases.

The reconstruction after surgical removal of the tumor is established according to the general principles of reconstruction, directed healing, suture, skin grafts, local flaps and microsurgical flaps can be used depending on the size, location and composition of the loss of substance, the age of the patient and the general condition of the patient [53].

In a prospective study [54] on reconstruction after neck and facial melanoma resection for 42 patients, an ordinal scale was used to evaluate several outcome criteria of reconstruction (pain, itching, color , scarring, stiffness, thickness, and irregularity) and a visual analogue scale (VAS) was used to assess the perception of appearance and patient satisfaction and the results were for patients who received skin grafts reported significantly adverse EVA scores compared to other reconstruction methods $(\mathrm{P}=0.046)$.

In addition, skin grafts received significantly less good ordinal scale assessments for itching $(\mathrm{P}=0.043)$, color $(\mathrm{P}=$ 0.047), scars $(\mathrm{P}=0.003)$ and stiffness $(\mathrm{P}=0.041)$ compared to other reconstruction methods.

The author of this study [54] concluded that the degree of emotional impairment after the face or neck reconstruction melanomas is correlated with the perception of the patient's appearance is more important from the patient's point of view. On the other hand, according to the present study, flaps are the preferred reconstruction methods, while skin grafts are the least favored. 
In another study done in 2014 by Hayashi T [55] whose objective was to analyze the post-excision reconstruction modes of the melanomas of the face, it was pointed out that the flaps were frequently used in the cases of large areas loss of substance with thick melanoma whereas skin grafts have been used for a wide variety of tumor thicknesses and for elderly patients with thick melanomas (due to the general conditions of the patients). This study concluded that the procedure for reconstruction after excision which is based on three important factors( size of loss of substance, severity of of a face melanoma and age of the patient) and that the reconstruction procedure had no impact on the prognosis.

For foot-plant melanoma, studies are being done to investigate the methods of repairing the substance loss after the excision and they concluded that the skin flap can provide well-vascularized local tissue for weight-bearing areas where a graft skin alone may not be appropriate as long as these flaps need to be well padded, which provides durable coverage especially for medium-sized post-excisional losses in acrolentiginous melanoma [54].

In general, for lower extremity melanomas and studies in this setting, the skin flap was found to be a reliable option but without impacting the survival of these patients.

For our population, The mode of repair of the tumor or post-tumor loss of operated patients: 6 patients have benefited from a direct suture, 26 patients have benefited from a cutaneous graft (of which 20 patients presented a good evolution and 4 patients had lysis of the transplant) whereas only one patient had a good neuro-sural type flap, from these results, we note that $81 \%$ of the cases of our series have benefited of a skin graft of which $77 \%$ had a good plastic evolution.

\section{Immunotherapy with:-}

1. Ipilimumab is a monoclonal antibody that binds to CTLA-4 and thus blocks its ability to regulate T-cell activation, proliferation, and effector function; it has demonstrated improved progression-free survival in multicenter international randomized trials. for patients with unresectable or advanced melanoma, leading to approval by the US Food and Drug Administration (FDA) in 2011.

2. Anti-PD-1 and PD-L1: The PD-1 pathway is a key immuno-inhibitory mediator of T cell depletion, blockage of this pathway can lead to $\mathrm{T}$ cell activation and expansion with enhanced effector functions and is marketed as Pembrolizumab.

3. Lambrolizumab received accelerated approval in 2014 demonstrating durable responses for patients whose disease had progressed after receiving ipilimumab.

4. Interleukin-2 (IL-2): High-dose interferon alpha-2b was approved by the US Food and Drug Administration in 1995 for the adjuvant treatment of patients with melanoma who have undergone complete surgical resection but who are considered to be at high risk of relapse (stage IIB, IIC, and III), however, prospective multicenter randomized trials have demonstrated that high-dose interferon and pegylated interferon improve non-relapse survival but improve not overall survival.

\section{Targeted therapy:-}

Studies to date indicate that BRAF and MEK inhibitors may have a significant impact on the natural history of melanoma, even though they do not appear to be curative as simple agents.

The BRAF inhibitor marketed in two products: Vemurafenib, a selective BRAF kinase inhibitor approved by the US Food and Drug Administration in 2011, has shown improved progression-free survival and overall survival in patients With advanced metastasis, its indication is limited to patients with a BRAF V600E mutation demonstrated by an approved test [57]. The second commercialized product is Dabrafenib which is a selective inhibitor of BRAF, it is a small molecule available orally and which was approved by the US Food and Drug Administration in 2013, it showed improved progression-free survival comparing it to chemotherapy. [56]

Vemurafenib is generally well tolerated, and its most common side effects are arthralgia, rash, alopecia, photosensitivity, fatigue, nausea, diarrhea and pruritus, so regular monitoring of liver enzymes is recommended since an elevation is possible. In case of adverse effects, symptomatic treatment may be started with a dose adjustment of vemurafenib. [56]

The MEK inhibitor: marketed under the name Trametinib is a selective inhibitor of MEK1 and MEK2 in the form of a small oral molecule approved by the US Food and Drug Administration in 2013 for inoperable patients or in case 
of 'a metastatic melanoma with BRAF V600E, he demonstrated improved progression-free survival by comparing it to chemotherapy. [56]

\section{Chemotherapy:-}

It can be used to treat advanced melanoma but it is not often used as first line treatment for other teams in the world because new forms of immunotherapy and targeted drugs have become available in these countries, it does not is usually as effective in melanoma as in some other types of cancer, but can relieve symptoms or prolong survival for some patients.

Several chemotherapy drugs can be used to treat melanoma: dacarbazine (also called DTIC), temozolomide paclitaxel, carmustine, cisplatin, carboplatin, and vinblastine.

Palliative treatment (radiotherapy):

Although melanoma is a relatively radiation-resistant tumor, palliative radiotherapy may relieve symptoms and retrospective studies have shown that symptom relief and some tumor shrinkage under radiation can occur in patients with multiple brain metastases. , bone metastases or compression of the spinal cord. [58]

The main progressive risk of melanoma is the occurrence of metastases, 43 patients (47.8\%) died because of melanoma evolution and the median survival after lymph node recurrence was 31.8 months ([CI] 23.3 to 40.3 months) [59].

The period between excision of the primary tumor and subsequent reoccurrence of recurrence is called metastatic dormancy and for melanomas, a period of dormancy may end with the emergence of a recurrence at the level of a metastatic site and rarely at the site of the primary tumor, so melanomas as much as some other cancers such as prostate and certain types of breast cancer, often have a very long cycle in which metastases do not manifest that after years or even decades, clinically localized melanoma can recur after an interval of 10 years or more [60].

The first non-contiguous sites at which melanoma cells are thought to spread are lymph nodes [59] with a significant risk for patients with melanoma> $1 \mathrm{~mm}$ thick, the presence of melanoma cells in the lymph nodes is the strongest predictor of recurrence and survival in patients, and if it is positive (sentinel lymph node biopsy) it is possible that tumor cells have already had access to the systemic circulation $[61 ; 62]$

The lungs and pleura are the most common sites of visceral metastasis in melanoma and one in ten melanoma patients will develop lung metastases at some point during their illness and the lungs are often the first site of visceral metastasis in melanoma, a series of autopsies reveal that more than $85 \%$ of patients with late-stage melanoma have evidence of lung metastases [63].

For brain metastases, The analyzes suggested that 20 to $54 \%$ of melanoma deaths result from brain metastases. At autopsy, 36 to 54\% of patients with metastatic melanoma have cerebral metastases, although relatively less than 12 to $20 \%$ have clinically evident metastases during their disease although when brain metastases are present, metastases visceral to other sites are also usually present. [32]

Hepatic metastases are clinically detected in $10-20 \%$ of patients with cutaneous melanoma, and subclinical liver metastases are much more common because they occur in $54-77 \%$ of patients with melanoma at the time of Melanoma is easy to detect and at low risk of spreading when diagnosed early, and its inducing factors (sun exposure) are manageable and is therefore an ideal tumor to organize prevention.

In our study, patients come at a late stage (39\% in stage III and IV) with a Breslow index and a high Clark level and nodal or visceral metastases which reflects the time elapsed between onset of lesions and the timing of the consultation and which can be explained by the lack of effective means of screening and prevention of patients at risk in our population hence the need to implement more effective ways to improve the prevention of melanoma.

\section{Conclusion:-}

The incidence of melanoma is rising worldwide. In Morocco, melanoma ranks third, after primary carcinomas and sarcomas, and affects the elderly with an average age of 60 years and a predominance of women. Foot localization is the most common estimated at $70 \%$ according to our study. 
Ultraviolet exposure remains the most incriminated factor in the development of melanomas, as well as other factors, the most important of which is the light phototype and the personal and family history of melanoma or other malignant skin tumors.

Surgical excision of lesions at the locoregional stage, is the optimal treatment in $80 \%$ of cases that confirms the diagnosis of melanoma and determine the thickness of Breslow and specify other factors histo-prognostic.

The interest of sentinel lymph node biopsy is highly evolved in the diagnosis of occult lymph node metastases.

For metastatic melanoma, many treatments have been attempted to improve the survival of patients of this stage, ipilimumab and inhibitors of B-raf (Vemurafenib and Dabrafenib) and MEK inhibitors (Trametinib) are the main options to metastatic disease since they are the only ones to have emonstrated survival gains and the choice of therapy should be individualized to take into account different factors such as BRAF mutation status, age, condition patient and the extent of the disease.

\section{Competing Interests:-}

The authors declare no competing interests.

\section{References:-}

1. K. Stoitchkov et T. Le Bricon : Marqueurs biologiques actuels de progression du mélanome cutané.Annales de biologie clinique 2000; 8 (2):157-65

2. Dereure.Mélanomes malins cutanés.Objectif Peau 1994 : 24-28.

3. Michèle M. Delaunay.Mélanome cutané: le diagnostic précoce, un devoir d'efficacité.La revue du praticien 2004; 54: 1179-1180.

4. Jean-luc Bulliard, Brian Cox. Cutaneous malignant melanoma in New Zealand: trends by anatomical site 19691993.International Journal of Epidemiology 2000; 29: 416-423

5. Laurence Chérie-Challine, Jean-Michel Halna et Laurent Remontet.

6. Situation épidémiologique du mélanome cutané en France et impact en termes de prévention. Bulletin Epidémiologique Hebdomadaire 2004 ; 2: 5-8.

7. Bellut, I. Vivard, R. Unvois et al. Nouveaux cas de mélanomes en Lorraine en 2000 et 2001 : enquête prospective sur deux ans réalisée par l'Union régionale des médecins libéraux de Lorraine. Nouv Dermatol $2003 ; 22$ : 154-157.

8. S. El Mesbahi, K. Zhouhair, T. El Ouzzani, S. Azzouzi, H. Lakhdar. Mélanome malin à casablanca : une tumeur à ne pas méconnaître ; Service de dermatologie, vénérologieCHU Ibn Rochd- Casablanca. Journal du praticien 2005 ; tome XIV supplément : 21.

9. L. Benzekri, R. Chraibi, J. Bouhlab, H. Chraibi, K. Senouci, M. Ait ourghroul, B. Hassam. mLes cancers cutanés à Rabat ; Service de dermatologie CHU Ibn Sina Rabat.Journal du praticien 2005 ; tome XIV supplément : 202-206

10. F. Bennouna Biaz, M. Ait Ourghroui, Habib-Dine et al. Le mélanome au Maroc. Les nouvelles dermatologiques 1998; 17 (2) : 56-59.

11. Nina M. Fisher, julie V. Schaffer, Marianne Berwick et al. Breslow depth of cutaneous melanoma: impact of factors related to surveillance of the skin, including prior skin biopsies and family history of melanoma. $\mathrm{J}$ Am Acad dermatol 2005; 53 (3): 393-406.

12. J. Michel Halna, M. Grandadam, A. Buemi. Etude épidémiologique des cancers cutanés basée sur la population d'un département français de 1988 à 1996. Résultats du registre du cancer du Haut-Rhin. Nouv Dermatol 2000; $19: 48-56$

13. Mathiey Boniol, bruce K. Armstrong, Jean-françois doré. Variation in incidence and fatality of melanoma by season of diagnosis in New South Wales Ausrtralia. Cancer Epidemiol Biomarkers Prev 2006; 15 (3): $524-528$

14. American Cancer Society. Cancer Facts \& Figures 2015. Atlanta, Ga: American Cancer Society; 2015.The Office for National Statistics on request UK,

15. July 2013, disponiblesur:(http://www.ons.gov.uk/ons/search/index.html?newquery=cancer+registrations 15Wagner JD, Gordon MS, Chuang TY, Coleman JJ 3rd. Current therapy of cutaneous melanoma. Plast Reconstr Surg. 2001, 105: 1774-1799.

16. Zanetti R, Rosso S, Martinez C, et al.: Comparison of risk patterns in carcinoma and melanoma of the skin in men: a multi-centre case-case-control study. Br J Cancer 94 (5): 743-51, 2006. 
17. Neale RE, Forman D, Murphy MF, et al.: Site-specific occurrence of nonmelanoma skin cancers in patients with cutaneous melanoma. Br J Cancer 93 (5): 597-601, 2005

18. Nelemans PJ, Rampen FH, Ruiter DJ, et al.: An addition to the controversy on sunlight exposure and melanoma risk: a meta-analytical approach. J Clin Epidemiol 48 (11): 1331-42, 1995.

19. Elwood JM, Jopson J: Melanoma and sun exposure: an overview of published studies. Int J Cancer 73 (2): 198203, 1997. [PUBMED Abstract]

20. Gandini S, Sera F, Cattaruzza MS, et al.: Meta-analysis of risk factors for cutaneous melanoma: III. Family history, actinic damage and phenotypic factors. Eur J Cancer 41 (14): 2040-59, 2005

21. Bennouna Biaz F, Ait-Ourhrouil M, habib-Dine, Lakhdar H, El Guedari B,Souadka A et al. Le mélanome au Maroc. Nouv Dermatol 1998; 1 7:56-59

22. Cogliano VJ, Baan R, Straif K, et al. Preventable exposures associated with human cancers. J Natl Cancer Inst 2011;103:1827-39.

23. Boniol M, Autier P, Boyle P, et al. Cutaneous melanoma attributable to sunbed use: systematic review and meta-analysis. BMJ 2012;345:e4757 doi: 10.1136/bmj.e4757.

24. Diffey BL. A quantitative estimate of melanoma mortality from ultraviolet A sunbed use in the U.K. Br J Dermatol 2003;149:578-81.

25. Colantonio S, Bracken MB, Beecker J. The association of indoor tanning and melanoma in adults: Systematic review and meta-analysis. J Am Acad Dermatol 2014;70(5):847-857.

26. Bishop JN, Harland M, Bishop DT: The genetics of melanoma. Br J Hosp Med (Lond) 2006, 67 (6): 299-304,

27. Scherer D, Kumar R: Genetics of pigmentation in skin cancer, a review. Mutat Res 705 (2): 141-53, 2010

28. Olsen CM, Carroll HJ, Whiteman DC. Estimating the attributable fraction for melanoma: a meta-analysis of pigmentary characteristics and freckling. Int J Cancer 2010;127:2430-45

29. Goggins WB, Tsao H: A population-based analysis of risk factors for a second primary cutaneous melanoma among melanoma survivors. Cancer 97 (3): 639-43, 2003.

30. Begg CB, Orlow I, Hummer AJ, et al.: Lifetime risk of melanoma in CDKN2A mutation carriers in a population-based sample. J Natl Cancer Inst 97 (20): 1507-15, 2005

31. van der Leest RJ, Liu L, Coebergh JW, et al. Risk of second primary in situ and invasive melanoma in a Dutch population-based cohort: 1989-2008. Br J Dermatol 2012;167(6):1321-30.

32. Marghoob AA, Slade J, Salopek TG, et al.: Basal cell and squamous cell carcinomas are important risk factors for cutaneous malignant melanoma. Screening implications. Cancer 75 (2 Suppl): 707-14, 1995.

33. Rosenberg CA, Khandekar J, Greenland P, et al.: Cutaneous melanoma in postmenopausal women after nonmelanoma skin carcinoma: the Women's Health Initiative Observational Study. Cancer 106 (3): 654-63, 2006

34. Brandt A, Sundquist J, Hemminki K: Risk of incident and fatal melanoma in individuals with a family history of incident or fatal melanoma or any cancer. Br J Dermatol 165 (2): 342-8, 2011.

35. Hemminki $\mathrm{K}$, Zhang $\mathrm{H}$, Czene $\mathrm{K}$ : Incidence trends and familial risks in invasive and in situ cutaneous melanoma by sun-exposed body sites. Int J Cancer 104 (6): 764-71, 2003. Goldstein AM, Chan M, Harland M, et al.:

36. High-risk melanoma susceptibility genes and pancreatic cancer, neural system tumors, and uveal melanoma across GenoMEL. Cancer Res2006, 66 (20): 9818-28.

37. Cogliano VJ, Baan R, Straif K, et al. Preventable exposures associated with human cancers. J Natl Cancer Inst 2011;103:1827-39. Singh S, Nagpal SJ, Murad MH, et al.

38. Inflammatory Bowel Disease Is Associated With an Increased Risk of Melanoma: A Systematic Review and Meta-Analysis. Clin Gastroenterol Hepatol 2013 doi: 10.1016/j.cgh.2013.04.033

39. Dinh QQ, Chong AH: Melanoma in organ transplant recipients: the old enemy finds a new battleground. Australas J Dermatol 48 (4): 199-207, 2009

40. Olsen CM, Knight LL, Green AC. Risk of melanoma in people with HIV/AIDS in the pre- and post-HAART eras: a systematic review and meta-analysis of cohort studies. PLoS One 2014;9(4):e95096.

41. A. Phan, S. Touzet,* S. Dalle, S. Ronger-Savle', B. Balme and L. Thomas. Acrallentiginous melanoma: histopathological prognostic features of 121 cases. British Journal of Dermatology 20071 57, pp311 -31 8

42. Kaskel P, Kind P, Sander S, Peter RU, Krahn G (2000) Trauma and melanoma formation: a true association. Br J Dermatol; 143:749-53. Les mélanomes malins cutanés : aspects épidémiologiques, clinques et thérapeutiques. Expérience du service de chirurgie plastique au CHU Mohammed VI de Marrakech - 147 -

43. Sanlorenzo M, Wehner MR, Linos E, et al. The Risk of Melanoma in Airline Pilots and Cabin Crew: A Metaanalysis.. JAMA Dermatol 2015;151(1):51-8. 
44. Dos Santos Silva I, De Stavola B, Pizzi C, et al. Cancer incidence in professional flight crew and air traffic control officers: Disentangling the effect of occupational versus lifestyle exposures. Int J Cancer 2012.

45. Gun RT, Pratt N, Ryan P, et al.: Update of mortality and cancer incidence in the Australian petroleum industry cohort. Occup Environ Med 63 (7): 476-81, 2006.

46. Zhang YP, Chu RX, Liu H. Vitamin A intake and risk of melanoma: a meta-analysis.. PLoS One 2014 Jul;9(7):e102527.

47. Grob JJ, Bonerandi JJ. The 'ugly duckling' sign: identification of the common characteristics of nevi in an individual as a basis for melanoma screening. Arch Dermatol 1998;134(1):103-104.

48. Vestergaard ME, Macaskill P, Holt PE, Menzies SW. Dermoscopy compared with naked eye examination for the diagnosis of primary melanoma: a meta-analysis of studies performed in a clinical setting. Br J Dermatol. Sep 2008;159(3):669-7

49. Balch CM, Gershenwald JE, Soong SJ, et al. Final version of 2009 AJCC melanoma staging and classification. J Clin Oncol. 2009;27(36):6199-6206.

50. Tran KT, Wright NA, Cockerell CJ. Biopsy of the pigmented lesion-when and how. J Am Acad Dermatol. 2008;59(5):852-871.

51. A. Lavie, C. Desouches, D. Casanova, J. Bardot, J.-J. Grob,R. Legré, G. Magalon. Mise au point sur la prise en charge chirurgicale du mélanome

52. National Comprehensive Cancer Network. NCCN Clinical Practice Guidelines in Oncology (NCCN Guidelines): Melanoma, version 1.2013.

53. Jecan C.R. Raducu L. Filip I.Hernik D. A REVIEW OF FREQUENT CUTANEOUS MALIGNANCIES University of Medicine "Carol Davila" Bucharest Department of Plastic and Reconstructive Microsurgery, Clinical Emergency Hospital "Prof. dr. Agrippa Ionescu” Bucharest RoJCED 2014; 1(1):46-51

54. Donald Buck, II, MD, Vinay Rawlani, MD, Jeffrey Wayne, MD, Gregory A Dumanian, MD, Robert Galiano, MD, and John YS Kim, Cosmetic outcomes following head and neck melanoma reconstruction: The patient's perspective, MD Division of Plastic and Reconstructive Surgery, Feinberg School of Medicine, Northwestern University, Chicago, Illinois, USA Plast Surg. 2012 Spring; 20(1): e10-e15.

55. Hayashi T1, Furukawa H, Oyama A, Funayama E, Saito A, Yamamoto Y.

56. An analysis of cheek reconstruction after tumor excision in patients with melanoma. the Department of Plastic and Reconstructive Surgery, Graduate School of Medicine, Hokkaido University, Sapporo, Japan.2014;25(2):e98-101.doi: 10.1097/SCS.0000000000000397.*

57. National cancer institute. Melanoma Treatment (PDQ®) November 7, $2014 \quad$ Disponible sur(http://www.cancer.gov/cancertopics/pdq/treatment/melanoma

58. Chapman PB, Hauschild A, Robert C, et al.:

59. Improved survival with vemurafenib in melanoma with BRAF V600E mutation. N Engl J Med 364 (26): $2507-$ 16, 2011. /HealthProfessional/page4)

60. American Cancer Society. Cancer Facts \& Figures 2015 available on (http://www.cancer.org/cancer/skincancermelanoma/detailedguide/melanoma-skin-cancer-key-statistics)

61. Jean-Emmanuel Bibault, Sylvain Dewas, Xavier Mirabel, Laurent Mortier, Nicolas Penel, Adjuvant radiation therapy in metastatic lymph nodes from melanoma, Academic Radiotherapy Departement, CLCC Oscar Lambret Comprehensive Cancer Center, Lille-Nord de France University, LILLE, France 6 February 2011

62. Shen, P.; Guenther, J.M.; Wanek, L.A.; Morton, D.L. Can elective lymph node dissection decrease the frequency and mortality rate of late melanoma recurrences? Ann. Surg. Oncol. 2000,

63. Nathanson, S.D. Insights into the mechanisms of lymph node metastasis. Cancer 2003, 98, 413-423.

64. Kingham, T.P.; Panageas, K.S.; Ariyan, C.E.; Busam, K.J.; Brady, M.S.; Coit, D.G. Outcome of patients with a positive sentinel lymph node who do not undergo completion lymphadenectomy. Ann. Surg. Oncol. 2010, 17, 514-520.

65. Balch, C.M.; Houghton, A.N.; Sober, A.J.; Soong, S. Cutaneous Melanoma, 4th edition; Quality Medical Publishing: St. Louis, MO, USA, 2003. 